\title{
Improving MAC Performance in Wireless Ad-Hoc Networks Using Enhanced Carrier Sensing (ECS)
}

\author{
Zhifei $\mathrm{Li}^{1}$, Sukumar Nandi ${ }^{2}$, and Anil K. Gupta ${ }^{1}$ \\ 1 School of Computer Engineering, Nanyang Technological University, \\ Singapore, 639798 \\ \{pg03802331, asgupta\}@ntu.edu.sg \\ 2 Dept. of Computer Science \& Engineering, Indian Institute of Technology, \\ Guwahati, India, 781039 \\ sukumar@iitg.ernet.in
}

\begin{abstract}
In IEEE 802.11, whenever a node detects an erroneous frame (e.g., a sensing range frame) on the medium, it defers the transmission by a fixed duration (represented by EIFS). We show that this duration is sometimes smaller and sometimes larger than the desired period by which the transmission should be deferred, and it leads to substantial unfairness and throughput degradation. We propose an enhanced carrier sensing (ECS) scheme, which distinguishes among the type of the erroneous frames based on their lengths and defers the transmission accordingly. Simulation results show that the ECS improves the fairness as well as the throughput substantially.
\end{abstract}

\section{Introduction}

Recently, wireless ad-hoc networks have attracted considerable research interest as they are easy to deploy and maintain. The Distributed Coordination Function (DCF) in IEEE 802.11 [4] is a CSMA/CA based protocol and is popularly adopted as the MAC protocol for ad-hoc networks. In the IEEE 802.11-based multi-hop wireless ad-hoc networks, since all the nodes share a common medium, only one flow among the contending flows can transmit during a certain duration. Therefore, whenever a frame exchange sequence between two nodes is in progress, it is extremely important to guarantee that all the other nodes in the interference range should defer their own transmission. In IEEE 802.11, whenever a node detects a physical carrier on the medium, it does not transmit. This is known as the physical carrier sensing. Moreover, even after the medium becomes idle, the node may need to defer further to allow the transmission of the remaining frames in the same sequence. This is known as the virtual carrier sensing (VCS). Under VCS, every frame will carry a duration value indicating the time by which the overhearing nodes should defer. It is clear that the VCS requires that all the potentially interfering nodes should overhear the ongoing frame transmission clearly, which may not be true due to wireless transmission 
errors and due to the fact that the carrier sensing range is normally greater than the transmission range. Therefore, an important issue is that whenever a node detects an erroneous frame, how long should the node defer its transmission?

In the current IEEE 802.11, whenever a node detects an erroneous frame on the medium, it always defers the transmission by a fixed duration indicated by the Extended Inter-Frame Space (EIFS) constant. We show that in some situations, the EIFS value is too large compared to the desired value, while in some other situations the EIFS value is too small. Respectively, we refer to these two cases as (i) large-EIFS problem, and (ii) small-EIFS problem. The two problems, together, are referred as the imprecise-EIFS problem. When smallEIFS problem occurs, a node may begin its transmission even though some other nodes in the interference range are still transmitting, resulting in collisions. On the other hand, when the large-EIFS problem occurs, the medium may be unnecessarily idle and the node may experience unfairness. Therefore, the imprecise-EIFS problem leads to immense unfairness and throughput degradation. In order to solve the imprecise-EIFS problem, we propose an enhanced carrier sensing $(\mathrm{ECS})^{1}$ mechanism in which the EIFS value is made variable in an adaptive manner. The simulation results show that our ECS greatly improves the fairness as well as the capacity utilization.

In the literature, there have been a lot of research-work $[1,2,9,10]$ focusing on improving fairness or throughput by modifying the contention avoidance $(\mathrm{CA})$ or contention resolution (CR) in the CSMA/CA-based protocols. However, to the best of our knowledge, this is the first work that focuses on the carrier sensing (CS) part to improve the performance of the CSMA/CA-based MAC protocols.

The rest of the paper is organized as follows. The imprecise-EIFS problem and the ECS are discussed in sections 2 and 3, respectively. The performance of ECS is studied in Section 4. Section 5 concludes the paper.

\section{Imprecise-EIFS Problem in IEEE 802.11}

\subsection{Preliminaries}

A special characteristic of wireless propagation is the attenuation of the transmission power over the distance traversed by the signal. Based on the attenuation, two ranges are defined: the transmission range (TR) and the sensing range (SR). Normally, the SR range is greater than the TR range [11]. Correspondingly, we call a frame detected by a node within TR as the TR frame, and a frame detected by a node out of TR but within SR as the SR frame. If there is no collision or transmission error, a node can receive a TR frame correctly. On the contrary, an SR frame can be detected by the carrier sensing but it cannot be received correctly, and therefore will be treated as an error. If capture is not allowed, a collision occurs whenever there are multiple frames (which may be a mixture of TR and SR frames) around a receiving node and all the frames will

${ }_{1}$ The concept of ECS was introduced in [7], where only the unfairness is considered for specific topologies. In this paper, the ECS is extended and generalized. 
be destroyed. On the contrary, if capture is allowed, one of the multiple frames may be captured (i.e., received correctly). In this work, we assume that capture is not supported.

To cope with the hidden-terminal problem, IEEE 802.11 defines a four-way handshaking, where a sequence of Request To Send (RTS), Clear To Send (CTS), Data, and Acknowledgement (ACK) frames, is transmitted for the transmission of every single data packet. For the convenience, we call the exchange of RTS/CTS/Data/ACK frames as a frame exchange sequence (FES). FES(X, Y) represents a FES between nodes X and Y, initiated by node X.

We now describe the simulation environment. NS-2 with CMU wireless extensions [3] is used for the simulations. For each single-hop flow, a Constant Bit Rate (CBR) traffic generates 200 packets per second. Each packet is 1000-bytes long, resulting in a traffic source rate of $1.6 \mathrm{Mbps}$. The raw bandwidth is set with $2 \mathrm{Mbps}$, leading to a maximum throughput about $1.4 \mathrm{Mbps}$ due to the overhead in IEEE 802.11. Static routing is used. Mobility and transmission errors are not explicitly considered in the simulation. The sensing range (SR) is 550 meters whereas the transmission range (TR) is 250 meters. Other system parameters are set according to the Direct Sequence Spread Spectrum (DSSS) [4], i.e., Slot-Time: $20 \mu s$; SIFS: $10 \mu s$; DIFS: $50 \mu s$; EIFS: $364 \mu s$.

\subsection{Large-EIFS Problem}

To explain the large-EIFS problem, scenario shown in Figure 1 is used, where the distance between two neighboring nodes is 200 meters. Therefore, nodes A and $\mathrm{C}$ are out of TR but within the SR of each other. As there are two singlehop flows, we expect that each flow should get a bandwidth about $0.7 \mathrm{Mbps}$. However, in the simulation, we found that the flow from $\mathrm{B}$ to $\mathrm{C}$ gets about 1.15 Mbps whereas the other flow gets only about $0.25 \mathrm{Mbps}$, showing how much unfair the IEEE 802.11 is in such a simple scenario.

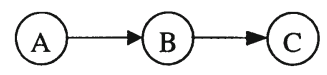

Fig. 1. 3-nodes with Two Single-hop Flows (Scenario-1)

We now explain the reason of the unfairness. Figure 2 demonstrates the process how a node defers its transmission while a FES between two other nodes is in progress. From Figure 2(a), it is easy to see that after a FES(A, B) is successfully completed, nodes A and B will start contending for the medium at about the same time. However, this is not true after the completion of a FES(B, C), as shown in Figure 2(b). Specifically, after node C sends an ACK to node B, node A will detect an SR frame and thus defer its transmission by EIFS rather than by DIFS as done at node B. Since EIFS is equal to SIFS + TxTime (ACK) $+D I F S$ [4], the deferment at node A is certainly of much longer duration than that at node $B$, resulting in unfairness. We call this large-EIFS problem because 
the EIFS value is larger than it should be to reflect the state of the medium. In fact, the EIFS value should be equal to DIFS in this case. To explain why the throughputs of the two flows differ so much, an analytical model has been developed, which validates the results here. Please refer to [8] for the model.

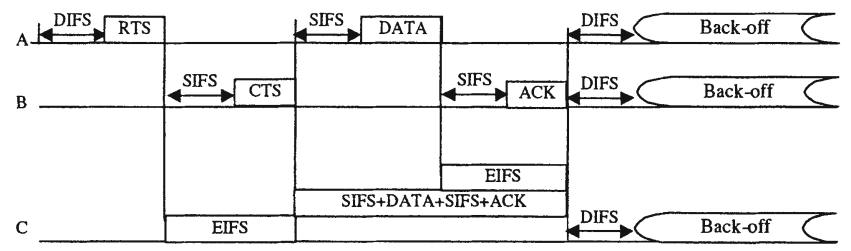

(a) FES (A, B) is in Progress while C Defers

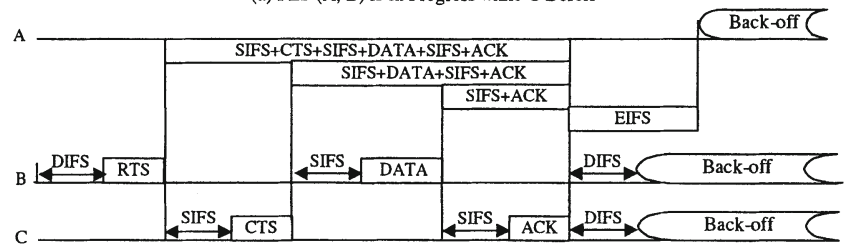

(b) FES $(B, C)$ is in Progress while A Defers

Fig. 2. Time Diagram Showing Frame Exchange Sequence

\subsection{Small-EIFS Problem}

Now we introduce the small-EIFS problem using the scenario of Figure 3. The distance between two neighboring nodes is again 200 meters. Our results show that the two flows share the bandwidth equally (in a long term). However, each of them only gets about $0.31 \mathrm{Mbps}$, resulting in an aggregate throughput about $0.62 \mathrm{Mbps}$, which is much smaller than the capacity (i.e., 1.4 Mbps).

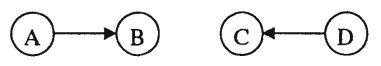

Fig. 3. 4-nodes with Two Single-hop Flows (Scenario-2)

To explain why the throughput degrades so much, let us consider the situation where nodes $\mathrm{C}$ and $\mathrm{D}$ are deferring their transmission, while $\mathrm{FES}(\mathrm{A}, \mathrm{B})$ is in progress. After node $\mathrm{B}$ sends a CTS to A, since node $\mathrm{D}$ is in the sensing range of node $\mathrm{B}$, node $\mathrm{D}$ gets an SR frame and defers its transmission with EIFS. Then, node $\mathrm{A}$ begins to send its Data frame to $\mathrm{B}$. However, since node $\mathrm{D}$ is out of the $\mathrm{SR}$ of $\mathrm{A}$, node $\mathrm{D}$ cannot detect this Data frame. Moreover, since the EIFS value is much smaller than the transmission time of the Data frame, after deferring for the EIFS duration, node D may begin transmit a frame even though node $\mathrm{A}$ is still transmitting the Data frame to $\mathrm{B}$. The two transmissions result in a collision at node B and thus node B will discard the Data frame. Likewise, due to 
symmetry in this topology, for a $\mathrm{FES}(\mathrm{D}, \mathrm{C})$, node $\mathrm{C}$ is also likely to discard the Data frame, explaining why the throughputs degrade so much. We call this as the small-EIFS problem because the EIFS value is smaller than it should be to reflect the state of the medium. In fact, in this situation, the EIFS value should be large enough to allow for the complete transmission of the Data frame.

In the above discussion, we assumed that the capture is not supported. When the capture is supported, node B may capture the Data frame from node A if the SNR of the frame is higher than a given threshold. Therefore, the throughputs will be greatly improved. However, when the capture is supported, the smallEIFS problem leads to unfairness as discussed in [8]. Therefore, we should resolve the small-EIFS problem irrespective of whether or not the capture is supported.

\subsection{General Scenario Involving Imprecise-EIFS Problem}

So far using specific examples, we have demonstrated how the large-EIFS and small-EIFS problems result in substantial unfairness and throughput degrade. In fact, a node will suffer from which specific type of problem depends upon what kind of frame it detects. This, in turn, depends upon the location of the node with respect to the location of the two nodes between whom the FES is in progress. For example, if a node gets an SR frame corresponding to a CTS, it may suffer from the small-EIFS problem. If a node gets an SR frame corresponding to an ACK, it may suffer from the large-EIFS problem. However, if a node gets an SR frame corresponding to a Data frame, the imprecise-EIFS problem does not arise because the EIFS value caters for the time needed for the next frame (i.e., ACK) to pass through. This is also true when an SR frame corresponding to a RTS is detected, since the next frame is a CTS frame, and the length of the CTS is equal to an ACK. Figure 4 shows a generalized scenario with impreciseEIFS problem where FES(A, B) is in progress. In the figure, $\mathrm{T}$ (frame) denotes a $\mathrm{TR}$ frame and $\mathrm{S}$ (frame) denotes an SR frame. We classify the entire region into seven areas. Nodes within different areas get different type of frames and thus may suffer from different type of problems. Note that in the area 6 , a node will not suffer from the small-EIFS problem after detecting an SR frame of CTS, since the node can also detect the SR frame corresponding to the Data.

\section{Enhanced Carrier Sensing (ECS)}

EIFS based deferment, as mentioned, is designed to allow the next frame in a FES to pass through. Since the next frame can be of any type (i.e., CTS, Data, or ACK), the transmission time of this frame may differ substantially. However, the IEEE 802.11 does not distinguish among different SR frames and uses the same constant EIFS value in all the cases, resulting the imprecise-EIFS problem. Therefore, if somehow a node can distinguish among different type of SR frames and adopts different value of EIFS accordingly, the problem can be greatly reduced and thus the performance (i.e., fairness and throughput) can be improved. In the light of this discussion, we make two proposals: (1) Whenever 

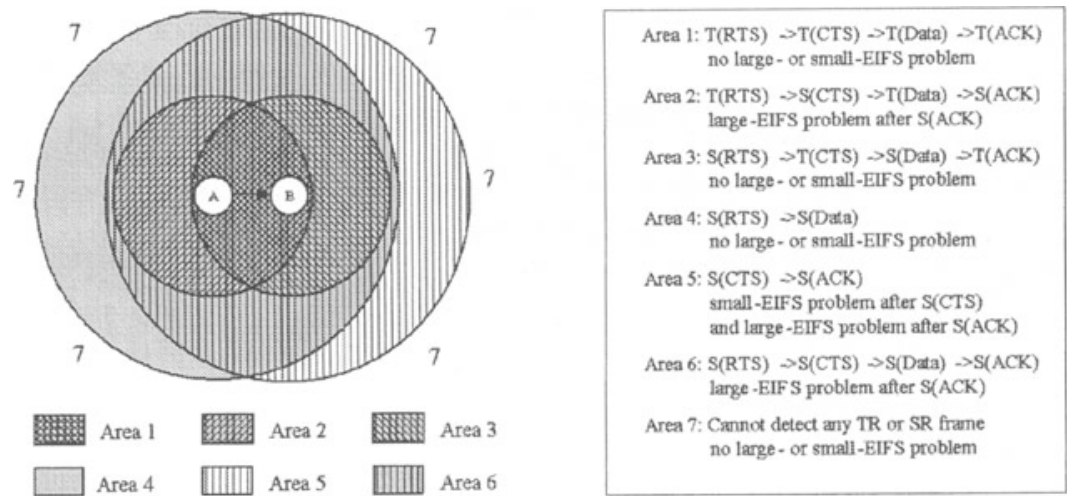

Fig. 4. Problems Arising in Different Areas while FES(A, B) is in Progress

a node detects an SR frame, it should try to identify the type of that frame; (2) EIFS value should be directly linked to the type of SR frame detected.

Since our CS mechanism tries to identify the type of an SR frame rather than just sensing its presence, we call it Enhanced Carrier Sensing (ECS).

\subsection{Distinguishing among SR Frames}

Basically, there are two methods in which we can distinguish among different type of SR frames. The first method is to use the recent history of frames observed on the medium to decide the type of the current SR frame as the frames are transmitted in a specific sequence. However, this method is very complex and the recent history may not provide very precise information as there may be multiple FESs in progress at any given time. The second method is to get the information from the frame itself. However, it is not trivial as the node cannot interpret the contents of an SR frame. We propose that the various type of frames should have different lengths, and based on the length of an SR frame observed on the medium, the type of the frame is identified.

We first need to differentiate the lengths of different type of frames. In IEEE 802.11 [4], the lengths corresponding to the control frames are as follows, RTS: 20 bytes, CTS: 14 bytes, ACK: 14 bytes. On the other hand, the header of a Data frame is 34 bytes, implying that the length of a Data frame must be greater than 34 bytes. In order to distinguish between CTS and ACK, the size of CTS should be increased by a few bytes. The reason why we increase the size of CTS rather than ACK is that the extra bytes in the CTS may be used by the receiver to add some receiver status information, which may be useful to the sender before it sends out the Data frame. What is an appropriate number of bytes that should be added to the CTS depends upon the trade-off between the sensitivity of the physical layer and the additional overhead introduced due to these bytes. In our implementation, we change the length of the CTS to 17 bytes. 
When a node gets an SR frame, to identify the type of the frame, the node only needs to detect the length of that frame. Before discussing how to detect the length, we have to introduce the format of the physical layer frame in the IEEE 802.11 [4]. As presented in Figure 5, a Physical Layer Convergence Protocol (PLCP) frame includes three parts: PLCP Preamble, PLCP Header, and MAC Protocol Data Unit (PDU). The PLCP preamble is used by the receiver to synchronize, while the PLCP header contains information to help the receiver to decide the end of the frame. The MAC PDU corresponds to the MAC layer frame, e.g., a RTS frame.

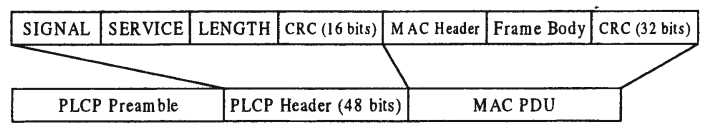

Fig. 5. PLCP Frame Format

Now we discuss how to detect the length (in terms of bytes) of an SR frame by considering two possibilities. The first possibility is that the PLCP header part of the frame can be correctly received by the physical layer but the MAC PDU part cannot be interpreted by the MAC layer. This is very likely due to the following two reasons. (i) In the IEEE 802.11, the PLCP header is always transmitted at the lowest transmission rate (i.e., $1 \mathrm{Mbps}$ ) while the MAC PDU in the same PLCP frame may be transmitted at a higher rate (e.g., $2 \mathrm{Mbps}$ or even higher). Generally, the Bit Error Rate (BER) under the case of lower transmission rate should be smaller if the Signal to Noise Ratio (SNR) is assumed to be the same, which should be true during the transmission of an entire PLCP frame. Therefore, the BER for the PLCP header may be much smaller than that for the MAC PDU. (ii) Moreover, the length of the PLCP header (i.e., 6 bytes) is much smaller than that of the MAC PDU (e.g., 20 bytes for a RTS frame). Due to the above two reasons, it is very likely that the PLCP header is correctly received while the MAC PDU is not. In such a situation, the MAC frame length (and thus the type) can be easily calculated from the fields contained in the PLCP header. Note that the above discussion also applies for a TR frame containing transmission errors as the PLCP header of a TR frame should always be interpretable.

The second possibility is that, when an SR frame is detected, even the PLCP header part in the PLCP frame cannot be correctly received by the physical layer. In such a situation, to identify the type of the MAC frame, we can make use of the Clear Channel Assessment (CCA) mechanism [4]. Specifically, based on the length of the time duration that the CCA mechanism indicates a busy medium, if the transmission rate is fixed for all the frames, the MAC layer can calculate the length (in terms of bytes) and thus identify the type. When the transmission rate of the MAC PDUs varies, as discussed in [8], the type of an SR frame can 
also be identified with the help of CCA mechanism. However, for the simplicity, in our simulation the transmission rate of the MAC PDU is fixed.

While our ECS will get help from the physical layer to detect the type of a frame, it does not need any enhancement at the physical layer. Therefore, it can be easily incorporated into IEEE 802.11.

\subsection{EIFS Values in ECS}

Based on the type of the frame observed on the medium, an EIFS value should be chosen accordingly. The basic rule for deciding the EIFS value is that it should be large enough to allow the complete transmission of the next frame in the sequence. Based on this rule, five different values of EIFS are defined in Figure 6. For a RTS type SR frame, the EIFS value is equal to SIFS + TxTime (CTS). When a node detects a CTS type SR frame, since the next frame in the sequence is a Data frame whose length may be variable and cannot be detected from the CTS type SR frame, we simply set the EIFS according to the maximum length allowed for the Data frames, i.e. Max-Data-Length. For a Data type SR frame, the EIFS value is set to SIFS + TxTime (ACK). Lastly, for an ACK type SR frame, since this is the last frame in the sequence, the EIFS value is set to DIFS.

In wireless ad-hoc networks, due to the mobility and dynamic propagation characteristics (e.g., fading), when an SR frame is detected by a node, it may only be a part of a frame rather than being a complete frame. We call this partialframe problem. Another type of problem may occur as follows. In the multi-hop scenario, spatial reuse of the bandwidth is very likely. Therefore, multiple frames may be there around a node at any given instant, resulting in a collision. As a result, the SR frame that a node detects may be an overlapped of several frames rather than a single frame. We call this overlapping-frames problem. In fact, it is very difficult for an MAC protocol to fully take care of these two problems. Therefore, whenever any of these two problems occur, as shown by the last line in Figure 6, we use the same value of EIFS as defined in the standard [4].

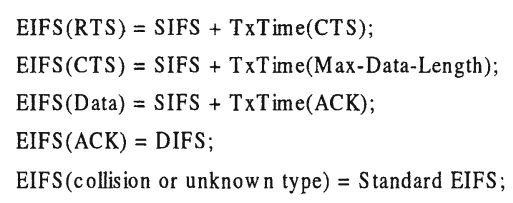

Fig. 6. Different EIFS Values in ECS

\section{Simulation Results}

In the performance evaluation of our Enhanced Carrier Sensing (ECS), the same simulation parameters are used as described in Section 2. 


\subsection{Performance for Scenarios with Two-Flows}

Scenario-1: This scenario (Figure 1) has been used to demonstrate the largeEIFS problem in Section 2. The average throughputs under the scenario are presented in Table 1. The IEEE 802.11 is very unfair due to the large-EIFS problem at node A. However, under our ECS, the two flows share the bandwidth equally. Moreover, as shown in Table 1, the aggregate throughput under the ECS is slightly greater than that under IEEE 802.11. This can be explained as follows. Consider that after a successful transmission by node B, nodes A and B contend for the medium. Suppose that the node $A$ generates a random back-off timer equal to 10 slots, while the node $B$ generates a random back-off timer with 30 slots, the node A will certainly win the contention in this round. If the large-EIFS problem does not occur as in the case of ECS, the medium will be idle for only 10 slots and then node A begins to transmit. On the contrary, if the large-EIFS problem occurs as in the case of IEEE 802.11, the medium will be idle by an extra duration equal to $(E I F S-D I F S)$, i.e., 16 slots. In summary, since the ECS solves the large-EIFS problem, it reduces the idle time of the medium, explaining the throughput improvement in ECS. Moreover, the throughput improvement should be much higher if a high-rate physical layer is used.

Scenario-2: This scenario (Figure 3) has been used to demonstrate the small-EIFS problem in Section 2. The average throughputs are presented in Table 2. Under the IEEE 802.11, due to the small-EIFS problem, the aggregate throughput is very small (i.e., $0.621 \mathrm{Mbps}$ ). However, under our ECS, the aggregate throughput greatly improves (i.e., 1.334 Mbps) as the ECS solves the small-EIFS problem. Note that the results are obtained under static routing and without capture capability. In [6], by varying the factors including the capture ability and the routing protocols, we have conducted an extensive simulation study for this topology.

Table 1. Throughput under Scenario-1

Table 2. Throughput under Scenario-2

\begin{tabular}{|c|c|c|}
\hline Throughput (Mbps) & IEEE 802.11 & ECS \\
\hline A to B & 0.254 & 0.705 \\
\hline B to C & 1.154 & 0.718 \\
\hline Aggregate & 1.408 & 1.423 \\
\hline
\end{tabular}

\begin{tabular}{|c|c|c|}
\hline Throughput (Mbps) & IEEE 802.11 & ECS \\
\hline A to B & 0.314 & 0.662 \\
\hline D to C & 0.307 & 0.672 \\
\hline Aggregate & 0.621 & 1.334 \\
\hline
\end{tabular}

Scenario-3: Now we discuss the results for the scenario shown in Figure 7. This scenario is similar to scenario-2 except that the direction of the flows is reversed. It is easy to see that after node A sends back an ACK frame to node $\mathrm{B}$, node $\mathrm{C}$ suffers from the large-EIFS problem if IEEE 802.11 is used. This is also true for node $B$ after the node $D$ sends back an ACK frame to node $\mathrm{C}$. The average throughputs of the two flows are presented in Table 3 . In contrast to scenario-1, the large-EIFS problem does not result in long-term unfairness in this scenario (i.e., otherwise the flows will have different average throughputs). However, it results in the short-term unfairness. To show this, in Figure 8, we present the Jain's index results using the sliding window method 
discussed in [5]. Generally, the higher the index, the fairer the protocol is. It is easy to see that the ECS greatly improves the fairness compared to IEEE 802.11. The reason that the large-EIFS problem leads to short-term unfairness is as follows. Consider that node B successfully transmits an upper-layer packet, and the chance that the node $\mathrm{B}$ gets control of the medium again is larger than that of node $\mathrm{C}$, as node $\mathrm{C}$ suffers from the large-EIFS problem. On the other hand, once node $\mathrm{C}$ controls the medium, node $\mathrm{C}$ will also have high chance to transmit consecutively. Therefore, the large-EIFS problem results in short-term unfairness. However, since the large-EIFS problem occurs at nodes B and $\mathrm{C}$ with the same possibility, the long-term fairness between the two flows is ensured.

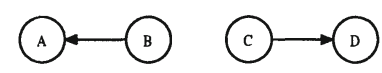

Fig. 7. 4-nodes with Two Single-hop Flows (Scenario-3)

Table 3. Throughput Comparison for Scenario-3

\begin{tabular}{|c|c|c|}
\hline Throughput (Mbps) & IEEE 802.11 & ECS \\
\hline B to A & 0.708 & 0.719 \\
\hline C to D & 0.702 & 0.710 \\
\hline Aggregate & 1.410 & 1.429 \\
\hline
\end{tabular}

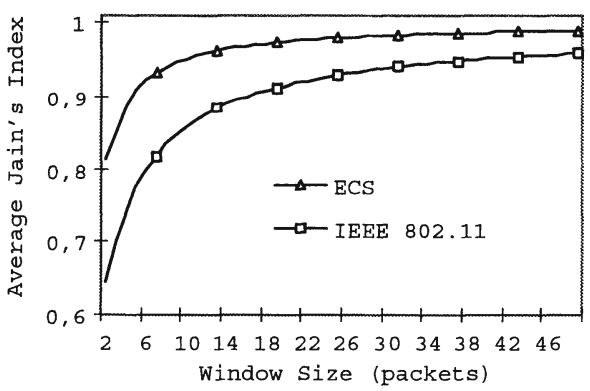

Fig. 8. Fairness Index Comparison for Scenario-3

Scenario-4: Now we discuss the results for the scenario shown in Figure 9, which is similar to scenario-2 except that the distance between nodes B and C is increased to 400 meters. Therefore, nodes $\mathrm{B}$ and $\mathrm{C}$ are out of the TR but within the SR of each other, and they suffer from the small-EIFS problem as indicated by area 5 in the generalized scenario (Figure 4). Table 4 presents the throughput results. The aggregate throughput under the IEEE 802.11 is $0.155 \mathrm{Mbps}$, which 
is much smaller than that under the ECS (i.e., $0.578 \mathrm{Mbps}$ ). However, even in the ECS, the aggregate throughput is still small compared to the medium capacity (i.e., about 1.4 Mbps). However, this is not due to the deficiency of ECS. For a detailed reason, please refer to [8].

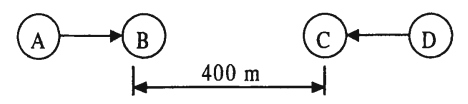

Fig. 9. 4-nodes with Two Single-hop Flows (Scenario-4)

Table 4. Throughput Comparison for Scenario-4

\begin{tabular}{|c|c|c|}
\hline Throughput (Mbps) & IEEE 802.11 & ECS \\
\hline A to B & 0.079 & 0.290 \\
\hline D to C & 0.076 & 0.288 \\
\hline Aggregate & 0.155 & 0.578 \\
\hline
\end{tabular}

Scenario-5 and -6: Here we present the results for two more scenarios. The topologies are presented in figures 10 and 11, respectively, while the throughputs are presented in tables 5 and 6, respectively. It is noted that the ECS improves both the fairness and the throughput. For a detailed explanation of these results, please refer to [8].

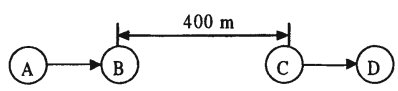

Fig. 10. Scenario-5

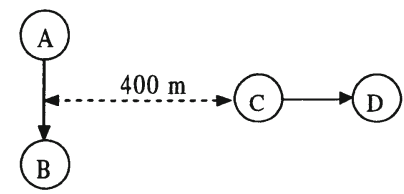

Fig. 11. Scenario-6

By referring to the generalized scenario discussed in Section 2.4, one can easily design some other scenarios (with two flows) to show the effects of the imprecise-EIFS problem and the advantage of our ECS [8]. However, due to space limitation, we do not present any more such scenarios here.

\subsection{Performance for a Complex Scenario}

Figure 12 shows the double-ring topology with 16 nodes. The distance between the sender and the receiver of each flow is 200 meters. The diameter of the inner circle is also 200 meters. Therefore, the diameter of the outer circle is 600 meters. The angle between any two neighboring flows is 45 degrees. The performance measures are presented in tables 7 and 8 . The ID of a flow is the same as the node ID of the flow's sender. The ECS greatly improves the aggregate throughput in this topology. As for the fairness, both the standard deviation and Jain's index under ECS are slightly greater than those under IEEE 802.11, 
Table 5. Throughput under Scenario-5 Table 6. Throughput under Scenario-6

\begin{tabular}{|c|c|c|}
\hline Throughput (Mbps) & IEEE 802.11 & ECS \\
\hline $\mathrm{A}$ to B & 0.0 & 0.075 \\
\hline $\mathrm{C}$ to D & 1.398 & 1.338 \\
\hline Aggregate & 1.398 & 1.413 \\
\hline
\end{tabular}

\begin{tabular}{|c|c|c|}
\hline Throughput (Mbps) & IEEE 802.11 & ECS \\
\hline $\mathrm{A}$ to B & 1.161 & 0.672 \\
\hline $\mathrm{C}$ to D & 0.254 & 0.766 \\
\hline Aggregate & 1.415 & 1.438 \\
\hline
\end{tabular}

which may convey very conflicting conclusions. If the standard deviation alone is considered, it seems that IEEE 802.11 is more fair than the ECS. On the other hand, the Jain's index is indicating that the ECS is more fair. Because the throughputs under the two schemes differ by an order of magnitude, a larger standard deviation does not imply that ECS is more unfair. Therefore, standard deviation is not always a good measure of fairness, while the Jain's index is.

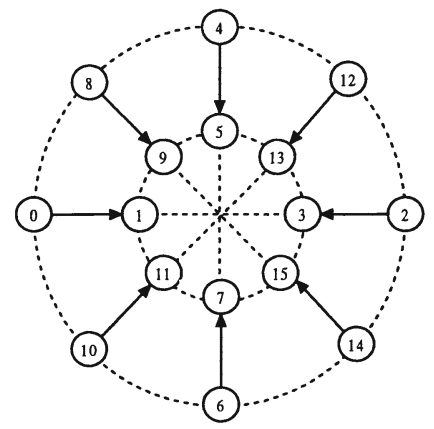

Fig. 12. Double-ring Topology: 16 Nodes with 8 Flows

Table 7. Throughput in Double-ring Topology

Table 8. Performance Measures

\begin{tabular}{|c|c|c|c|c|c|c|c|c|}
\hline Flow ID & 0 & 2 & 4 & 6 & 8 & 10 & 12 & 14 \\
\hline IEEE 802.11 & 0.0169 & 0.0214 & 0.0197 & 0.0195 & 0.0207 & 0.0206 & 0.0197 & 0.0209 \\
\hline ECS & 0.1586 & 0.1587 & 0.1542 & 0.1517 & 0.1641 & 0.1627 & 0.1663 & 0.1483 \\
\hline
\end{tabular}

\begin{tabular}{|c|c|c|c|}
\hline Metrics & Aggregate & STD DEV & Jain's Index \\
\hline IEEE 802.11 & 0.1594 & 0.0013 & 0.9957 \\
\hline ECS & 1.2646 & 0.0059 & 0.9986 \\
\hline
\end{tabular}

We also conducted the simulation for several more complex scenarios (e.g., chain and grid topologies) [8]. It is found that the ECS always improves the performance of IEEE 802.11.

\section{Conclusions}

In IEEE 802.11, whenever a node detects an erroneous frame, it will always defer by a fixed duration (represented by EIFS). Due to this fixed EIFS value, we showed that two problems arise: small- and large-EIFS problems, which lead to considerable unfairness and throughput degradation. To solve the problems, we have proposed an enhanced carrier sensing (ECS) mechanism. In the ECS, 
the lengths of the frames are made different. Based on the length of a frame observed on the medium, the type of the frame can be detected, and the node defers the transmission for a duration accordingly. In order to identify the length of an erroneous frame, the ECS utilizes the information provided by the physical layer. However, the ECS does not require any enhancement at the physical layer, and thus it can be easily incorporated into IEEE 802.11. The extensive simulation results have shown that the ECS eliminates the small- and large-EIFS problems to a great extent, and thus improves the fairness as well as throughput drastically.

\section{References}

1. V. Bharghavan, A. Demers, S. Shenker, and L. Zhang, "MACAW: A Media Access Protocol for Wireless LANs," ACM SIGCOMM, 1994.

2. F. Cali, M. Conti, E. Gregori, "Dynamic Tuning of the IEEE 802.11 Protocol to Achieve a Theoretical Throughput Limit," IEEE Journal on Selected Areas in Communications, December 2000, pp.785-799.

3. CMU Monarch Group. CMU Monarch Extensions to NS, http://www.monarch.cs.cmu.edu/.

4. IEEE, "Wireless LAN Medium Access Control (MAC) and Physical Layer (PHY) specifications," IEEE 802.11 standards, June 1999.

5. C.E. Koksal, H. Kassab, H. Balakrishnan, "An Analysis of Short-Term Fairness in Wireless Media Access Protocols," ACM SIGMETRICS, 2000.

6. Z.F. Li, S. Nandi, A.K. Gupta, "Study of IEEE 802.11 Fairness and its Interaction with Routing Mechanism," IFIP MWCN, 2003.

7. — "Improving Fairness in IEEE 802.11 using Enhanced Carrier Sensing (ECS)," accepted by IEE Proceedings on Communications, Dec., 2003.

8. - "Improving MAC Performance in Wireless Ad-hoc Networks using Enhanced Carrier Sensing (ECS)," full version of the paper, avaliable at http://www.ntu.edu.sg/home5/pg03802331/.

9. H. Lou, S. Lu, V. Bharghavan, "A New Model for Packet Scheduling in Multi-hop Wireless Networks," ACM MOBICOM, 2000.

10. T. Nandagopal, T. Kim, X. Gao, V. Bharghavan, "Achieving MAC Layer Fairness in Wireless Packet Networks," ACM MOBICOM, 2000.

11. K.X. Xu, M. Gerla, S. Bae, "How Effective is the IEEE 802.11 RTS/CTS Handshke in Ad Hoc Networks?", IEEE Globecom, 2002. 